\title{
SPATIALLY RESOLVED LIFETIMES IN EFG AND STRING RIBBON SILICON AFTER GETTERING AND HYDROGENATION STEPS
}

\author{
P. Geiger, G. Kragler, G. Hahn, P. Fath, E. Bucher \\ Universität Konstanz, Fachbereich Physik, Fach X916, 78457 Konstanz, Germany
}

\begin{abstract}
Lifetime improvement in EFG and String Ribbon silicon by gettering or defect passivation has been investigated by photoconductance decay. But in contrast to former studies measurements have been carried out in a spatially resolved way.

In this way the mapping techniques have been found to be indespensible for an accurate study of processing steps' impact on material quality. This is due to very strong lifetime variations that have been measured throughout the wafer (between $<1 \mu$ s and $>300 \mu \mathrm{s}$ ), and because areas of the same starting quality have turned out to react very differently on applied processing steps. Consequently, the results of integral measurements strongly depend on the nature of the areas incorporated in the specific sample. A statistical evaluation of the mapped lifetimes gives an impression of the uncertainties inherent to integral measurements.
\end{abstract}

\section{INTRODUCTION}

Silicon ribbons are grown directly out of the melt in the required thickness. Hereby wafer costs can be reduced as expensive wafering steps and related material losses are avoided. The most promising materials in this field to date are Edge-defined Film-fed Growth (EFG) silicon that is commercially produced by ASE Americas Inc. and String Ribbon silicon grown by Evergreen Solar Inc. which has just made the step from pilot line to industrial production. The specific crystal growth procedures of both materials result in grain structures and structural defect distributions which are significantly different from those in conventionally cast multicrystalline silicon. Solar cell processing consequently requires the development of processing schemes that are specifically adapted to the needs of these materials. One way of studying the impact of processing steps on the silicon ribbons is to measure minority charge carrier lifetimes before and after application of selected processing steps. Intensive work in this field has already been carried out using integral measurements [1,2]. But as the mentioned silicon ribbons show quite strong variations of material properties within a few square centimeters, it is not obvious that regions of different quality react in the same way and to the same extent to a certain processing step $[3,4]$. Therefore, we have investigated the impact of different processing steps on ribbon silicon in a spatially resolved way.

\section{EXPERIMENTAL APPROACH}

The minority charge carrier lifetimes of $5 \times 5 \mathrm{~cm}^{2}$ wafers were mapped using the technique of microwave induced photoconductance decay $(\mu-\mathrm{PCD})$. The measurements were performed under low injection conditions using a laser wavelength of $905 \mathrm{~nm}$ for carrier generation and bias light. The obtained lifetime values represent an effective lifetime $\tau_{\text {eff }}$ which can be regarded as the bulk lifetime if a sufficient surface passivation is provided during the measurement. This was realized by using a iodine-alcohol solution. Due to the resulting reproducible surface passivation it was possible to investigate the impacts and synergetic effects of different processing steps by measuring the corresponding changes in bulk lifetime $\tau_{\text {bulk }}$.

The determination of $\tau_{b u l k}$ from the decay of photoconductance requires the choice of an adequate time range during which the measurement system evaluates the decay. Because of strong lifetime variations within EFG and String Ribbon wafers it is not possible to select one single time range that is well-suited for the whole sample. As a consequence, usually not all values in $\mu$-PCD lifetime mappings of EFG or String Ribbon wafers are reliable. This problem was addressed by measuring each wafer partly or as a whole several times with different time ranges and finally combining them in an adequate way with the help of developed software procedures. Therefore, very high as well as rather low lifetime values are reliable in the mappings presented in this study.

Four different processing sequences have been investigated as illustrated in Fig. 1. In order to obtain comparable surfaces $20 \mu \mathrm{m}$

\begin{tabular}{|c|c|c|c|}
\hline Sequence 1 & Sequence 2 & Sequence 3 & Sequence 4 \\
\hline$\mu-\operatorname{PCD} 1$ & $\mu-\mathrm{PCD} 4$ & $\mu-\mathrm{PCD} 8$ & $\mu-P C D 12$ \\
\hline & P-diffusion & & P-diffusion \\
\hline & & Al-gettering & Al-gettering \\
\hline & Em. removal & BSF removal & Em. / BSF rem. \\
\hline & $\mu-\operatorname{PCD} 5$ & $\mu-\operatorname{PCD} 9$ & $\mu-P C D 13$ \\
\hline MIRHP-pass. & MIRHP-pass. & MIRHP-pass. & MIRHP-pass. \\
\hline$\mu-P C D 2$ & $\mu-\operatorname{PCD} 6$ & $\mu$-PCD 10 & $\mu$-PCD 14 \\
\hline Illumination & Illumination & Illumination & Illumination \\
\hline$\mu-\operatorname{PCD} 3$ & $\mu-\mathrm{PCD} 7$ & $\mu-\mathrm{PCD} 11$ & $\mu-P C D 15$ \\
\hline
\end{tabular}

Fig.1: Structure of the experiment. Spatially resolved bulk lifetime measurements have been performed after various successive processing steps.

were acidically etched back on each side of the wafers at the 
very beginning. Before each measuring step indicated in Fig. 1 the samples were chemically cleaned and their surface was passivated with an iodine alcohol solution. Emitter diffusion was performed in a quartz tube furnace and $2 \mu \mathrm{m}$ Aluminium for gettering were evaporated. Before subsequent measurements emitter as well as back surface field (BSF) were etched back in order to avoid recombination in the emitter region or problems with surface passivation on the back side during $\mu$-PCD measurement. Hydrogen passivation was realized with the help of a microwave induced remote hydrogen plasma (MIRHP) as described in [6]. Finally, the stability of this passivation could be examined after ten hours of illumination under one sun.

\section{RESULTS}

Whenever a $\mu$-PCD measurement is indicated in Fig. 1 several measurements were performed and combined as described in the previous section, so that the obtained mappings show reliable high and low lifetime values. The mappings of an EFG wafer that underwent sequence 2 are shown in Fig. 2 representatively. Due to an inadequate decay evaluation time range there is a region in mapping (a) where the measurement was not totally reliable. Those points, which are located in the best area of the mentioned mapping (at the centre of the bottom), were not taken into account and are marked grey. The left column of Fig. 2 shows the bulk lifetimes after each processing steps, whereas the right column contains mappings that illustrate the lifetime alterations caused by the single processing steps. Analysing pictures (a) and (b) or rather (b) - (a) one finds that phosphorous gettering leads to strong improvements of partly up to $100 \mu \mathrm{s}$ in areas of rather high starting lifetimes of seven or more microseconds. For areas of lower starting lifetimes enhancements of only $2 \mu$ s or even less have been detected. Hydrogenation, instead, improves most areas with a worse performance by about $20 \mu$ s on average. But areas with high starting lifetimes are effected again most strongly - in the dashed rectangle of mapping (c) bulk lifetimes of up to $300 \mu$ s have been measured. Illumination tests have revealed the MIRHP passivation to be stable under illumination within the range of measurement techniques accuracy.

A very important aspect visible in Fig. 2, however, is that regions of comparable as grown lifetimes exist within EFG or String Ribbon silicon (see Fig. 4) which are influenced differently by the individual processing steps. The marked region II of Fig. 2, for example, improves significantly during hydrogenation, whereas in region III lifetimes remain rather low, although it has shown the same starting lifetime and even slightly better lifetime values after P-gettering. Moreover, region I shows an area with about the same starting lifetime as in regions II and III, but this one has hardly improved during the whole processing sequence.

This ununiform behaviour can be seen more clearly in split mappings like those of a String Ribbon wafer given in Fig. 4 that also underwent processing sequence 2 of Fig. 1. In this graph the lifetime mapping performed before any processing step (a) has been split up into three sub-mappings: One with bulk lifetimes of up to $2 \mu \mathrm{s}$ (a1), another one covering values between 2 and $7 \mu \mathrm{s}$ (a2) and a last one with lifetimes above $7 \mu \mathrm{s}$ (a3). The corresponding columns (a1)... (d1) etc. illustrate the influence of processing steps on the areas of each lifetime range. Mappings (ax) show absolute starting lifetime values whereas (bx), (cx) and (dx) de-

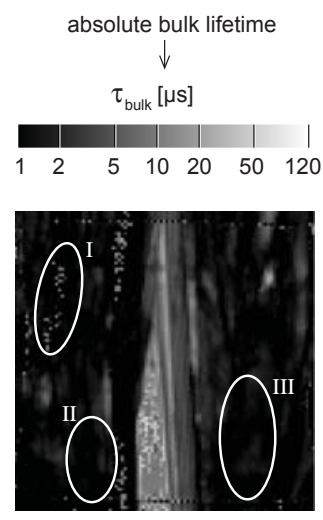

a) after defect etching

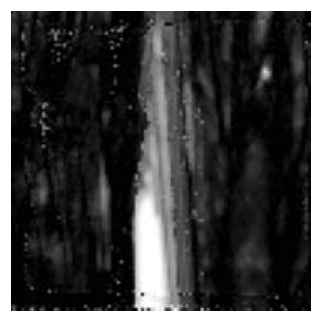

b) after P-diffusion

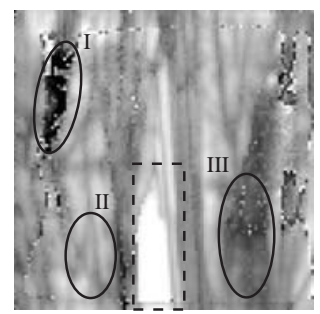

c) after MIRHP-pass

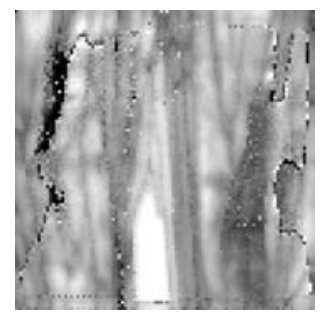

d) after illumination

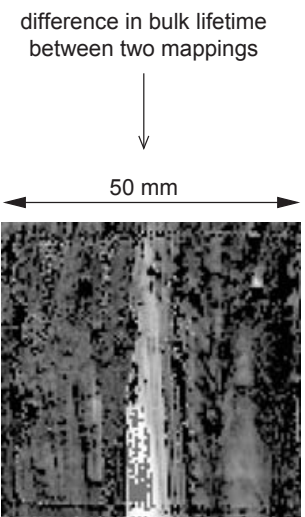

(b) $-(a)$
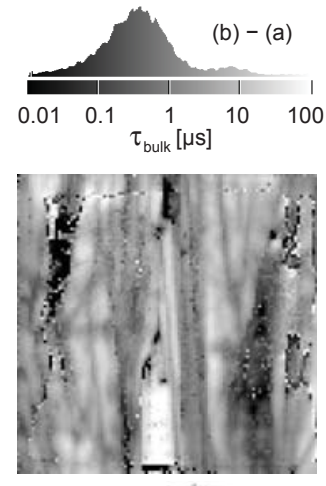

\section{(c) - (b)}
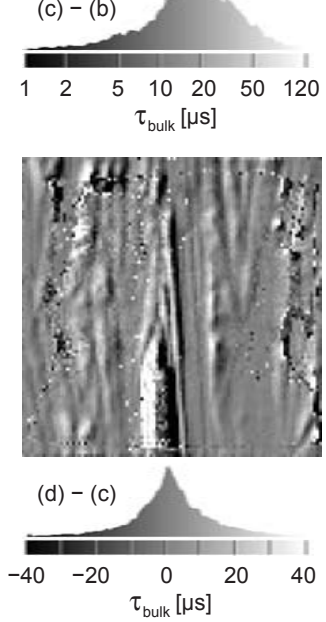

Fig.2: Bulk lifetime mappings of an EFG wafer corresponding to sequence 2 after appropriate combination of various measurements. The left column shows the bulk lifetimes measured after different processing steps with all mappings scaled in the same way. Differences between two steps are illustrated by the right column.

pict gains or losses caused by the different processing steps with respect to the lifetimes measured before the individual step. The grey points now indicate lifetime data that does not fulfil the criterion for a certain selection.

A comparison of the mappings in one column clearly shows that 
regions of comparable starting lifetime can react very differently on the applied processing steps. In each category examples of such areas have been marked to illustrate this.

Due to the existence of these regions of different behaviour and their inhomogeneous distribution according to usual wafer sizes, integral lifetime measurements appear not to be well suited for an accurate analysis. Instead, spatially resolved measurement techniques should be used for detailed lifetime investigations in EFG or String Ribbon Silicon. This conclusion is strengthened if one calculates the mean values and standard deviations for each sub-mapping. This has been done for the split mappings of all sequences given in Fig. 1. The results for String Ribbon silicon are shown in Fig. 3a together with the corresponding mean starting lifetimes of the sub-mappings. The bars look similar to those known from integral measurements. But the very large standard deviations illustrate that an enormous amount of information has been lost, despite of the fact that the measurements have already been split into groups of different starting lifetimes. As a consequence, it is difficult to compare the impact of the different processing steps with the help of the bars given in Fig. 3a. However, it can be clearly seen that remote hydrogen plasma passivation is much less efficient if no gettering step precedes the hydrogenation process.

The splitting of lifetime mappings has also been performed for the different EFG samples processed according to the sequences given in Fig. 1. Again in each category of starting lifetime areas of comparable quality can be found which react differently on the applied processing steps. The resulting bars in Fig. 3b show that spatially resolved measurement techniques should be used for accurate investigations also in the case of EFG silicon.

\section{SUMMARY}

Standing for the sum of spatially resolved investigations of different processing sequences the one of P-gettering followed by remote plasma hydrogen passivation has been discussed. It could be shown that P-gettering leads to strong improvements of bulk lifetime (partly more than $100 \mu$ s ) in regions of starting lifetimes of more than $7 \mu \mathrm{s}$. For areas with lower starting values an enhancement of only some microseconds has been measured. A following hydrogenation step, instead, improves also the worst regions quite properly. But again areas with rather high starting lifetimes are effected most strongly. However, it has been found that the remote plasma hydrogenation has a much less benefical influence if no gettering step precedes the passivation, whereas it does not matter whether P- or Al-gettering or both is performed. A discussion of the other investigated processing sequences can be found in $[7,8]$.

All spatially resolved measurements of minority charge carrier lifetimes in EFG and String Ribbon silicon have shown strong variations of bulk lifetimes within some square centimeters of wafer area. Those inhomogenities have revealed to become even more striking after gettering and hydrogen passivation. Moreover, it has been found that there exist regions of comparable starting lifetime within the mentioned materials, which react very differently to the various solar cell processing steps. Some improve very strongly reaching lifetimes of up to $300 \mu \mathrm{s}$, whereras others are only insufficiently enhanced. As such areas seem to be inhomogeneously distributed according to usual wafer sizes they influcence inte-
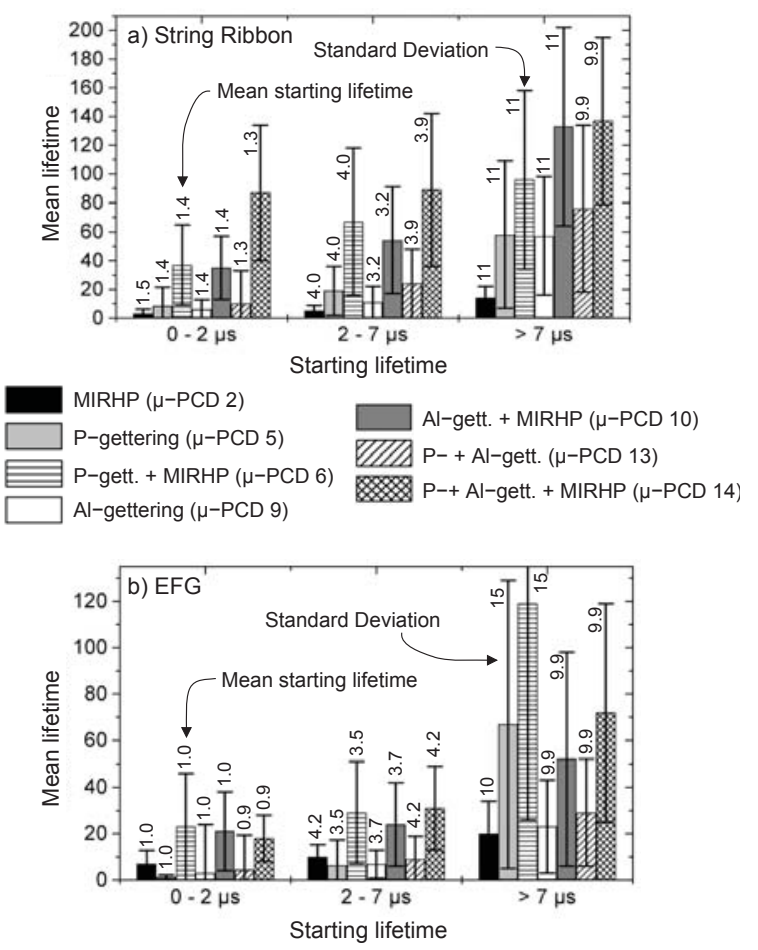

Fig.3: Mean lifetime values after different processing steps (see Fig. 1) calculated for regions of different starting lifetimes.

gral lifetime measurements. Consequently, spatially resolved measurement techniques are required for detailed analyses of EFG or String Ribbon silicon material.

\section{ACKNOWLEDGEMENT}

This work was partly supported within the KoSi programme by the German Bundesministerium für Wirtschaft under contract number 0329858J.

\section{REFERENCES}

[1] A. Rohatgi, V. Yelundur, J. Jeong, A. Ebong, D. Meier, A. M. Gabor, M. D. Rosenblum, Proc. 16th EC PVSEC, Glasgow, UK (2000) 1120.

[2] J.-W. Jeong, A. Rohatgi, M. D. Rosenblum, J. P. Kalejs, Proc. 28th IEEE PVSC, Anchorage, Alaska (2000) 83.

[3] P. Geiger, G. Hahn, P. Fath, E. Bucher, Proc. 16th EC PVSEC, Glasgow, UK (2000) 1214.

[4] P. Geiger, G. Hahn, P. Fath, E. Bucher, Sol. En. Mat. \& Sol. Cells 72, 2002, pp. 155-163.

[5] B. Bathey, J. Kalejs, M. Rosenblum, M. Kardauskas, R. Giancola, J. Cao, Proc. 28th IEEE PVSC, Anchorage, Alaska (2000) 194.

[6] M. Spiegel, P. Fath, K. Peter, B. Buck, G. Willeke, E. Bucher, Proc. 13th EC PVSEC Nice, France (1995) 421.

[7, 8] P. Geiger, G. Kragler, G. Hahn, P. Fath, E. Bucher, to be published in Proc. 17th EC PVSEC, Munich, Germany (2001). 


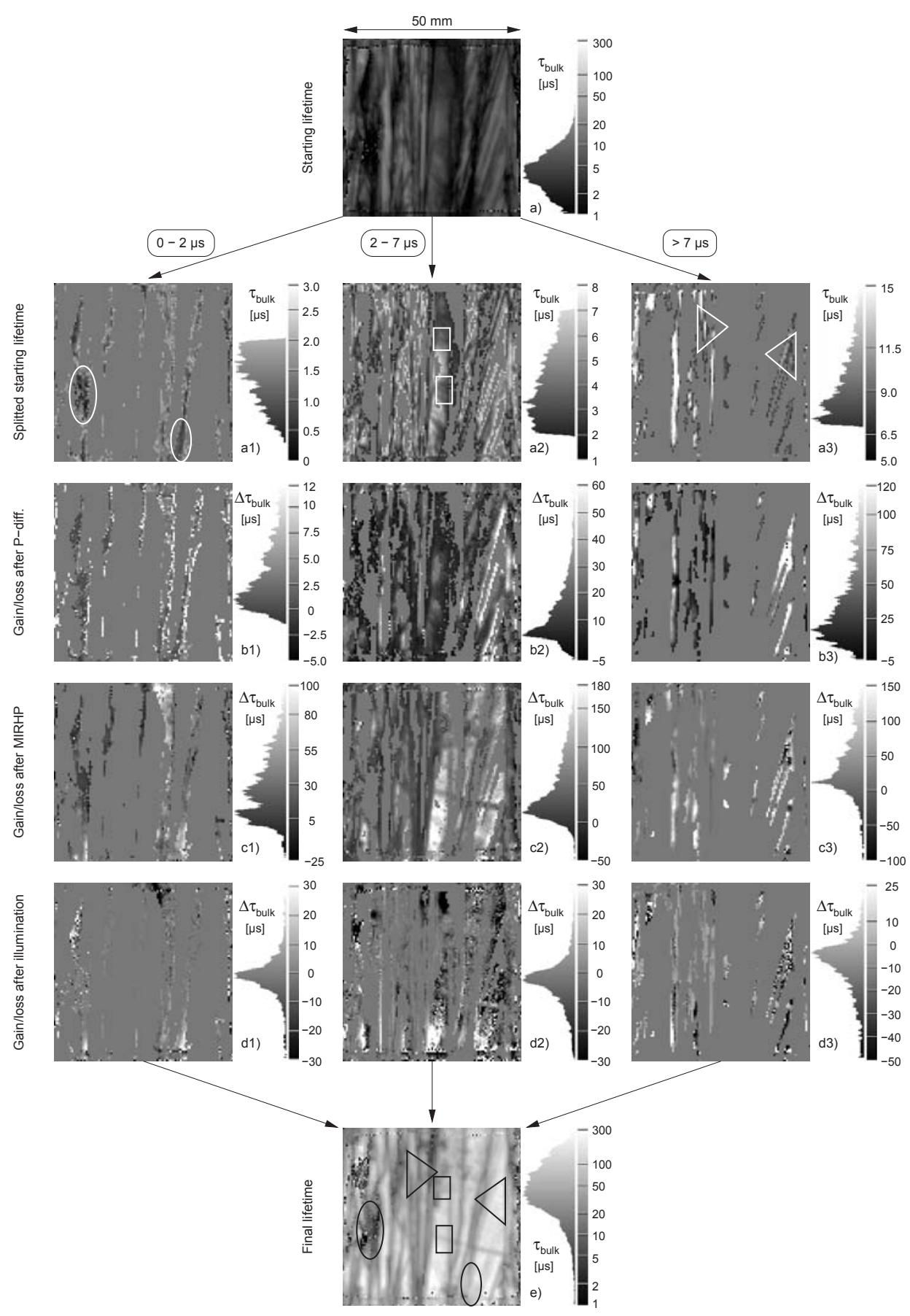

Fig.4: Bulk lifetime mappings of a String Ribbon wafer corresponding to sequence 2 as obtained after appropriate combination of various measurements. Mappings have been split according to their starting lifetimes. Graphs (a), (ax) and (e) show absolute lifetime values, whereas (bx), (cx) and (dx) illustrate gains or losses caused by each processing step as related to the lifetime values measured before this individual step. 